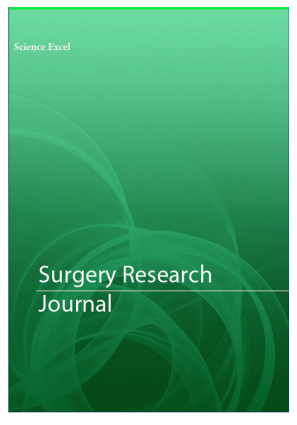

Correspondence

lu O Mikheiev

State Institution "Zaporizhia Medical Academy of Post-Graduate Education Ministry of Health of Ukraine", Ukraine Email: mikheev.u.a@gmail.com

- Received Date: 21 Nov 2021

- Accepted Date: 26 Nov 2021

- Publication Date: 14 Dec 2021

Keyword

Chronic Pancreatitis, Biliary Strictures.

Copyright

(c) 2021 Science Excel. This is an openaccess article distributed under the terms of the Creative Commons Attribution 4.0 International license.

\title{
Surgical Procedures for Biliary Stricture in Chronic Pancreatitis
}

\author{
Yareshko VG, Mikheiev lu O, Skrypko VD, Shpylenko OF \\ State Institution "Zaporizhia Medical Academy of Post-Graduate Education Ministry of Health of Ukraine", Ukraine
}

\begin{abstract}
Background: Biliary stricture (BS) in chronic pancreatitis (CP) is observed in up to $21 \%$ of patients with CP. However, there are no clearly established criteria when the one should operate in case of the CBD dilatation without increased liver enzymes. Attention is now paid to endoscopic interventions, the disadvantage of which is the need for repeated procedures, as well as less effectiveness in the treatment of pain in CP, while the operation can be aimed at various manifestations of CP. Early surgery - up to 3 years from onset of symptoms of $\mathrm{CP}$ showed improved results in terms of pain and exocrine function, but it is unknown whether it is of value in prevention of BS.

Goal. To determine the optimal timing of surgery to prevent biliary stricture in CP, the optimal type of intervention in the bile ducts, the optimal combination of surgery on the bile ducts and pancreas and to establish indications for the latter.

Materials and methods: Retrospective analysis of case histories of patients who were operated due to chronic pancreatitis from 2001 to 2020. Diagnostic criteria of BS were mechanical jaundice and/or dilatation of $\mathrm{CBD} \geq 10 \mathrm{~mm}$. BS was confirmed by intraoperative cholangiography (IOCG). Choledochoduodenostomy (CDS), hepatic and choledochoenterostomy (GEA / HEA), transduodenal papillosphincterotomy (TDPST), duodenum-preserving resections of the pancreatic head (DPRPH) were performed. The effectiveness of operations assessed by the absence of cholangitis / mechanical jaundice during observation. Statistical analysis was performed using IBM SPSS Version27. Pearson's $\chi^{2}$, Fisher's exact criterion, was used to analyze categorical data. The level of statistical significance is set at $\mathrm{p}<0,05$.

Results: No recurrence of BS achieved in $85.7 \%$ of patients. Recurrence of BS (cholangitis / jaundice) was observed in 8 patients (14.3\%). Signs of recurrence were found: in the group of TDPST in $33.3 \%$, in $16.7 \%$ of patients with CDS, in $9.7 \%$ of patients with DPRPH. For the latter, resection decompression was supplemented in these patients by fenestration of the choledochus into the resection cavity. BS was observed in $18.8 \%$ of patients with symptoms lasting up to 3 years and in $33.8 \%$ - more than 3 years. Conclusions: Surgery up to 3 years from the onset of symptoms of CP prevents the occurrence of BS. Dilatation of the choledochus $\geq 10 \mathrm{~mm}$ in a patient with $\mathrm{CP}$ indicates the presence of BS. In the case of an inflammatory mass or pseudocyst in the head of the pancreas as a cause of BS, it may be sufficient to eliminate it via DPRPH, in particular Frey's procedures. If external decompression of the CBD was not sufficient, the best operation is choledochoenteroanastomosis (GEA / HEA). In the absence of inflammatory mass in the head, it is also advisable to combine pancreatojejunostomy with GEA / HEA.
\end{abstract}

\section{Introduction \& Literature review}

AIn chronic pancreatitis (CP), the parenchyma of the gland is replaced by fibrous scar tissue, which can cause compression of the intrapancreatic part of the common bile duct. Pseudocysts, especially located in the head of the pancreas, can also cause compression and obstruction of the pancreas in rare cases. Clinically, biliary stricture (BS) in $\mathrm{CP}$ may be manifested by jaundice and / or cholangitis. Instrumental techniques such as ultrasound (US), computed tomography (CT), and magnetic resonance cholangiopancreatography (MRI) detect dilatation of the bile ducts and help to make a differential diagnosis, especially with malignant neoplasms of the periampullary area. Hyperbilirubinemia is less important in detecting, especially latent cholestasis, compared to elevated alkaline phosphatase (AL). In patients with $\mathrm{CP}$ and persistent elevation ( $\geq 1$ month) of LF, BS should be suspected and appropriate examinations should be performed. Given the possible development of secondary biliary cirrhosis of the liver as a result of prolonged cholestasis, biliary hypertension should be regarded as an independent indication for appropriate intervention. However, there is such question as whether to consider the expansion of FGM $\geq 10 \mathrm{~mm}$ in a patient with $\mathrm{CP}$ without biochemical signs of cholestasis, 
a sign of biliary stricture or a risk factor for the development of clinically significant BS in the future?

BS can be a manifestation of neglect of the fibroinflammatory process in the $\mathrm{CP}$, so it is important not only to eliminate it properly, but also to prevent the occurrence of BS. Much attention in the work of the last 5 years is paid to endoscopic methods of treatment of $\mathrm{CP}$, including $\mathrm{BS}$ in CP. At the same time, there are data indicating the need for surgery for pain in CP, after many endoscopic interventions and lower efficiency of such operations, due to the fact that the process in the $\mathrm{CP}$ becomes more neglected. Naturally, the question is it necessary to delay the operation in patients with CP arises? Do we need to perform a lot of stenting with BS, instead of an operation that can potentially resolve both $\mathrm{BS}$ and allows you to perform pathogenetic intervention on the $\mathrm{CP}$ ?

Varieties of possible operations for BS have their disadvantages and advantages in terms of short-term and long-term results, so you should determine the most optimal and universal method.

The frequency of obstruction of the biliary ducts among patients with CP varies from $13 \%$ to $21 \%$ [1], which emphasizes the urgency of the problem.

Recent studies have focused on the choice of stent (plastic or metal (SEMS)), and in the case of the metal one it is important to choose whether the stent is coated or not [2-7], there are also isolated reports of much rarer causes BG, such as the stone of the main pancreatic duct (GLP), which caused biliary obstruction [8]. The degree of success (resolution of stricture) and failure (stent migration, recurrence of stricture) and complications varies between studies. When using a fully coated metal stent (FCSEMS) that is a method considered $[2,9]$ as one that will get rid of the disadvantages inherent in plastic and uncoated metal stents (the need for many re-stenting for plastic [2] and germination of the stent for uncoated SEMS [7 ]) in 123 patients with benign BS in $81 \%$ of cases received a solution of stricture, of which recurrence of stricture occurred in $3(10 \%)$ patients with $\mathrm{CP}$, resolution of stricture in $27(90 \%)$ patients with $\mathrm{CP}$. Typical complications were migration $(9.7 \%)$, occlusion (4.9\%), cholangitis (4.1\%), acute pancreatitis (3.3\%). The authors came to the optimistic conclusion that although $\mathrm{CP}$ is a more difficult to stent etiology of BS, longer duration of the stent may improve the success of the procedure, but with the caveat that the published high success rate was achieved due to the fact that research centers were highflowing with experienced endoscopists. [2]. A less positive result was obtained in a single-center study of FCSEMS, and specifically it was the resolution of BS in $70.6 \%$ of patients with $\mathrm{CP}$, failures were associated with calcifications of the parenchyma of the pancreas in $80 \%$ [10]. The place of stenting in the treatment of BS in CP summarizes the international consensus on interventional endoscopy in $\mathrm{CP}$, so in patients with significant calcifications and / or inflammatory mass in the head of the pancreas, surgical treatment of strictures is the method of choice [11].

Much less work is devoted to the surgical treatment of BS in $\mathrm{CP}$, in particular the comparison of the surgical method and stenting, $[12,13]$ but with the important conclusion of the meta-analysis that in the treatment of BS caused by CP, surgical treatment can be not only a backup method after stenting failure. it can be considered as an effective first-line method that will save the patient from multiple repeated endoscopies [13].

The hypothesis that early surgical treatment, up to 3 years of symptom duration, is a decisive factor in pain control, has been confirmed in many studies [14-16]. If BS is considered a sign of a more severe disease [17], caused not only by fibrosis of the tissues around the choleda, but also by pseudocysts and GLP stones [18], it is possible that early surgery can not only improve pain control but also reduce the risk of symptomatic BS. Biliary stricture in many studies is diagnosed with an enlargement of the gastrointestinal tract $\geq 10 \mathrm{~mm}$ in a patient with $\mathrm{CP}$ in the presence of biochemical signs of cholestasis for a month or more [18], which is considered an indication for endoscopic or surgical resolution, due to the likelihood of secondary biliary cirrhosis [19]. At the same time, it is unknown whether to consider the expansion of FFS $\geq 10 \mathrm{~mm}$ in the absence of biochemical signs of cholestasis, a reason for appropriate simultaneous surgery to prevent BS, during CP surgery on pain control. The spread of non-invasive instrumental methods, such as endoscopic ultrasound and MRHPG, found that up to $33 \%$ of patients with dilatation of the liver at normal levels of liver enzymes have an objective cause that are choledocholithiasis, BS in chronic pancreatitis, periampullary diverticula. In the literature, the summary of "surgical treatment" is often used, although there is a fairly wide arsenal of operations on the biliary tract in BS. Each of these methods has its advantages and disadvantages, the ratio of which varies between studies. For example, some authors consider hepaticocholedoenterostomy (GEA / HEA) to be the "gold standard" [22-24], while others consider it sufficient to perform choledochoduodenoanastomosis (CDS) [25] or transduodenal papillosphincteroplasty (TDPST) that are regarded as a potential cause of recurrent cholangitis and a potential risk factor for cholangiocarcinoma [26]. The fenestration of the choledochus into the resection cavity is widely known, but some recent studies have shown a high recurrence rate of biliary obstruction with this technique [23]. Therefore, in addition to determining the benefits of surgical treatment of BS, it should be established whether all techniques will be accompanied by such benefits equally. One of the rational proposed combinations $[23,24]$ is Frey's procedure and, if necessary, $\mathrm{BDA}$.

\section{Materials and methods}

SA retrospective analysis of case histories for the period from 2001 to 2020 of patients operated on for CP, as well as for $\mathrm{CP}$ with complicated BS was performed. The criteria for the diagnosis of the latter were the clinic of mechanical jaundice, and / or imaging data for the expansion of hepatic duct $\geq 10 \mathrm{~mm}$. LF as a routine test was not performed in most patients. Using intraoperative cholangiography (IOCG) through the stump of the gallbladder, before and after DPRPH or cystopancreatojenostomy (CPEA) when the contrast in the duodenum immediately, the diagnosis of BS was excluded, when the contrast in the duodenum after DPRPH and CP. If the intervention on the CP was not sufficient, operations were performed on the bile ducts. In high-risk patients with coagulopathy and laboratory evidence of hepatic insufficiency, only BDA was performed, without $\mathrm{CP}$ intervention. Depending on the above options 
for operations performed, the following groups of patients are formed - 1) TDPST with \ without CP interventions; 2) hepaticoentero-, choledochoenterostomy (GEA / HEA); 3) duodenal-preserving resections of the head of the pancreas (DPRPH); 4) choledochoduodenostomy. The results of operations of patients with clearly defined duration of symptoms of CP (up to 3 years - "early surgery on the CP" or "Early surgery" and after 3 years - "late surgery on the $\mathrm{CP}$ ", respectively "Late surgery") were analyzed separately. The main criterion for the effectiveness of operations was the absence of episodes of cholangitis and / or mechanical jaundice during observation. The diagnostic criteria of the latter were complaints of fever, episodes of itchy skin, dark urine, yellowing of the sclera, detection of leukocytosis $\geq 10 \times 109$ increase in liver enzymes $\geq 2$ norms. A survey and periodic examination of patients operated before 2011 - during 2011, operated later - a year after surgery. Statistical analysis was performed using IBM SPSS Version 27 (SPSS Inc, Chicago, Illinois). Continuous and categorical data are given as standard deviations (JI) and absolute / relative frequencies, respectively. Pearson's $\chi^{2}$, Fisher's exact criterion, was used to analyze categorical data. The level of statistical significance is set at $\mathrm{p}<0.05$.

\section{Results}

In the analysis of data of 147 patients who underwent intervention on the pancreas (DPRPH, pancreatojejunoanastomosis), BS in patients of the group "Early Surgery" (74 persons) compared with patients of the group "Late Surgery" with clinical and laboratory signs of mechanical jaundice was detected in 13 patients (17.8\% of the group) and 17 patients (23.9\% of the group), respectively; BS without mechanical jaundice at the time of hospitalization was observed in 1 patient (1.4\%) and 7 patients $(9.9 \%)$, respectively, ie stricture confirmed by IOCG was observed in $18.8 \%$ in the group "Early Surgery" and in 33, $8 \%$ in the group "Late Surgery", $\mathrm{p}=0.04$. During the observation in both groups there were no new cases of benign BS. In the analysis of all patients with a previous diagnosis of "biliary stricture due to CP", 61 patients were identified, 5 were excluded due to suspicion or later established malignant nature of BS, remaining 56. As can be seen in table. 1, attacks of cholangitis or jaundice were observed in 8 patients (14.3\%). The worst results in the TDPST group - in 3 of 9 patients $(33.3 \%)$ there were signs of recurrence, cholangitis occurred in $2(16.7 \%)$ of 12 patients with CDU, as well as in $3(9.7 \%)$ of 31 a patient who underwent DPRPH. In the latter case, these three patients resected decompression was supplemented by fenestration of the choledochus into the resection cavity due to the existing but weak penetration of contrast into the duodenum during IOCG. There were no statistically significant differences in the ratio of parenchymal calcifications, GPP concretions, the presence of inflammatory mass in the pancreas (diameter of the pancreas $\geq 4 \mathrm{~cm}$ ) and the presence or absence of recurrence of jaundice / cholangitis by groups ( $\mathrm{p}>0.05)$.

There were two fatalities: 1 patient in whom Frey's operation was supplemented with CDU - died of hepato-renal failure, 1 patient in whom only DPRPH was performed - died of intra-abdominal bleeding. Of the early complications were noted: 1 patient from the group DPRPH - intra-abdominal bleeding stopped by relaparotomy and hemostasis, partial failure of BDA in 2 patients with CDU, and 1 patient with GEA $\backslash$ HEA - conservative treatment, biliary fistulas closed independently the average duration of fistula is 7 days, JI 2). The groups did not differ statistically significantly in early postoperative complications ( $\mathrm{p}>0.05)$.

\section{Discussion}

The success of surgical treatment (absence of recurrence) of biliary stricture was achieved in $85.7 \%$, which is comparable with the data of other authors [13,22]. Given the absence of recurrence in patients with hepatic, choledochoenteroanastomosis, the level of success could be even better with the routine implementation of this variant of BDA in all patients. We did not observe the development of cholangiocarcinoma in patients who underwent TDPST or CDU, but these patients had a fairly high incidence of cholangitis and jaundice, even higher than the literature data - in our series $33.3 \%$ and $16.7 \%$, respectively against $11.3 \%$ and $10.9 \%$, respectively [26].

Interestingly, there is a case of one of the patients who underwent Frey's operation in combination with TDPST in 2011. During the observation of this patient there was a recurrence of BS, manifested first by recurrent cholangitis, and later stricture Fater's papilla with episodes of choledocholithiasis that required subsequent 4 endoscopic papillosphincterotomy with lithoextraction. Subsequently, in 2021, the patient underwent hepaticoenteroanastomosis with a loop of the small intestine, which was previously used for PEA.

We did not establish a statistically significant relationship between recurrence of biliary stricture and calcifications of the pancreatic head, pancreatic stones, inflammatory mass in the head before surgery, which indicates in favor of surgical treatment of BS in the presence of such factors, which in turn are proven risk factors for endoscopic stenting [10,11].

The authors consider a reliable method of performing IOCG both to confirm the presence of BS and to determine the need to add to DPRPH hepatic or choledochoenteroanastomosis. Decompression of the intrapancreatic part of the choledoch by excision of the inflammatory mass was sufficient for 28 (90.3\%) of 31 patients for contrast to enter the duodenum, no signs of recurrence of BS in these patients were observed. Therefore, we support the methodology and conclusions of Merdrignac et al., And Ray, S et al. [23,24] who published a similar approach.

Studies on the term disease, as a decisive factor in pain control and preservation of pancreatic function [14,27], did not consider the likelihood of complications from the surrounding organs, such as biliary stricture. We found a statistically significant correlation between the duration of CP symptoms over 3 years and the higher incidence of BS. Given that in patients operated up to 3 years from the onset of symptoms, without BS at the time of surgery, we did not observe its occurrence in the future, we can assume that early surgery for CP can also prevent the development of local complications.

\section{Conclusions}

The development of biliary stricture in CP in our study was associated with the duration of the disease, ie with a disease 
duration of more than 3 years, the incidence of stricture was statistically significantly higher. This may be in favor of "early" surgery for $\mathrm{CP}$, without long-term conservative treatment, thus preventing complications such as BS.

According to abdominal imaging, the dilatation of the RV $\geq 10 \mathrm{~mm}$ is a sufficient reason to suspect BS, and accordingly to check its presence and the need for its correction with IOCG.

In the presence of an inflammatory mass or pseudocyst in the head of the CP, it may be sufficient to eliminate it DPRPH, in particular Frey's procedure, if according to IOCG external decompression of the choledochus was not sufficient, the best operation is to perform hepatic or choledochoenteroanastomosis (GEA) with the loop of the small intestine for $\mathrm{Ru}$, as for pancreatoenteroanastomosis (PEA). In the absence of inflammatory mass in the head, it is also advisable to combine PEA with GEA / HEA. Methods such as CDU, TDPST, and fenestration of the choledochus into the resection cavity during DPRPH are accompanied by a higher incidence of cholangitis and recurrence of jaundice, although this difference was not statistically significant in this study.

\section{References}

1. Ma MX, Jayasekeran V, Chong AK. Benign biliary strictures: prevalence, impact, and management strategies. Clin Exp Gastroenterol. 2019;12:83-92.

2. Saxena P, Diehl DL, Kumbhari V, et al. A US Multicenter Study of Safety and Efficacy of Fully Covered Self-Expandable Metallic Stents in Benign Extrahepatic Biliary Strictures. Dig Dis Sci. 2015;60(11):3442-3448.

3. Seicean A, Vultur S. Endoscopic therapy in chronic pancreatitis: current perspectives. Clin Exp Gastroenterol. 2014;8:1-11.

4. Nakai $\mathrm{Y}$, Isayama $\mathrm{H}$, Wang HP, et al. International consensus statements for endoscopic management of distal biliary stricture. J Gastroenterol Hepatol. 2020;35(6):967-979.

5. Leong RW. Metallic Stents for Benign Extrahepatic Biliary Stricture: In Praise of Self-Expansion?. Dig Dis Sci. 2015;60(11):3158-3159.

6. Ohyama H, Mikata R, Ishihara T, et al. Efficacy of multiple biliary stenting for refractory benign biliary strictures due to chronic calcifying pancreatitis. World J Gastrointest Endosc. 2017;9(1):12-18.

7. Bartel MJ, Higa JT, Tokar JL. The Status of SEMS Versus Plastic Stents for Benign Biliary Strictures. Curr Gastroenterol Rep. 2019;21(7):29.

8. Shetty AJ, Pai CG, Shetty S, Balaraju G. Pancreatic Calculus Causing Biliary Obstruction: Endoscopic Therapy for a Rare Initial Presentation of Chronic Pancreatitis. Dig Dis Sci. 2015;60(9):2840-2843.

9. Haapamäki C, Kylänpää L, Udd $M$, et al. Randomized multicenter study of multiple plastic stents vs. covered selfexpandable metallic stent in the treatment of biliary stricture in chronic pancreatitis. Endoscopy. 2015;47(7):605-610.

10. Perri V, Boškoski I, Tringali A, et al. Fully covered self-expandable metal stents in biliary strictures caused by chronic pancreatitis not responding to plastic stenting: a prospective study with 2 years of follow-up. Gastrointest Endosc. 2012;75(6):1271-1277.

11. Kitano M, Gress TM, Garg PK, et al. International consensus guidelines on interventional endoscopy in chronic pancreatitis.
Recommendations from the working group for the international consensus guidelines for chronic pancreatitis in collaboration with the International Association of Pancreatology, the American Pancreatic Association, the Japan Pancreas Society, and European Pancreatic Club. Pancreatology. 2020;20(6):10451055.

12. Udd M, Kylänpää L, Kokkola A. The Role of Endoscopic and Surgical Treatment in Chronic Pancreatitis. Scand J Surg. 2020;109(1):69-78.

13. Huszár O, Kokas B, Mátrai P, et al. Meta-Analysis of the Long Term Success Rate of Different Interventions in Benign Biliary Strictures. PLoS One. 2017;12(1):e0169618.

14. Issa Y, Kempeneers MA, Bruno MJ, et al. Effect of Early Surgery vs Endoscopy-First Approach on Pain in Patients With Chronic Pancreatitis: The ESCAPE Randomized Clinical Trial. JAMA. 2020;323(3):237-247.

15. Yang CJ, Bliss LA, Freedman SD, et al. Surgery for chronic pancreatitis. Pancreas. 2015;44(5):819-23.

16. Bouwense SAW, Kempeneers MA, van Santvoort HC, Boermeester MA, van Goor H, Besselink MG. Surgery in Chronic Pancreatitis: Indication, Timing and Procedures. Visc Med. 2019;35(2):110-118.

17. Cauchy F, Regimbeau JM, Fuks D, Balladur P, Tiret E, Paye F. Influence of bile duct obstruction on the results of Frey's procedure for chronic pancreatitis. Pancreatology. 2014;14(1):2126.

18. Hyun JJ, Irani SS, Ross AS, Larsen MC, Gluck M, Kozarek RA. Incidence and Significance of Biliary Stricture in Chronic Pancreatitis Patients Undergoing Extracorporeal Shock Wave Lithotripsy for Obstructing Pancreatic Duct Stones. Gut Liver. 2021;15(1):128-134.

19. Kleeff J, Whitcomb DC, Shimosegawa T, et al. Chronic pancreatitis. Nat Rev Dis Primers. 2017;3:17060.

20. Smith I, Monkemuller K, Wilcox CM. Incidentally Identified Common Bile Duct Dilatation: A Systematic Review of Evaluation, Causes, and Outcome. J Clin Gastroenterol. 2015;49(10):810-815.

21. Bruno M, Brizzi RF, Mezzabotta L, et al. Unexplained common bile duct dilatation with normal serum liver enzymes: diagnostic yield of endoscopic ultrasound and follow-up of this condition. J Clin Gastroenterol. 2014;48(8):e67-e70.

22. Ray S, Ghatak S, Das K, et al. Surgical Management of Benign Biliary Stricture in Chronic Pancreatitis: A Single-Center Experience. Indian J Surg. 2015;77(Suppl 2):608-613.

23. Ray S, Das K, Jana K, Das R, Kumar D, Khamrui S. Frey Procedure Combined with Biliary Diversion for the Treatment of Chronic Pancreatitis-Related Biliary Obstruction: Impact of the Types of Diversion. World J Surg. 2020;44(7):2359-2366.

24. Merdrignac A, Bergeat D, Rayar $M$, et al. Frey procedure combined with biliary diversion in chronic pancreatitis. Surgery. 2016;160(5):1264-1270.

25. Leppard WM, Shary TM, Adams DB, Morgan KA. Choledochoduodenostomy: is it really so bad?. J Gastrointest Surg. 2011;15(5):754-757.

26. Tocchi A, Mazzoni G, Liotta G, Lepre L, Cassini D, Miccini M. Late development of bile duct cancer in patients who had biliary-enteric drainage for benign disease: a follow-up study of more than 1,000 patients. Ann Surg. 2001;234(2):210-214.

27. Ke N, Jia D, Huang W, et al. Earlier surgery improves outcomes from painful chronic pancreatitis. Medicine (Baltimore). 2018;97(19):e0651. 